\title{
1974 - Markierung einer Wende Kommentar zu Lucius Burckhardts „Wer plant die Planung?“
}

Kommentare von: Wolf Reuter, Beate Binder, Jens S. Dangschat, Monika Grubbauer, Michael Guggenheim, Stadt von Unten, Iris Dzudzek

Der referenzfreie und bestechend unprätentiöse Duktus, mit dem Lucius Burckhardt seine Gedankengänge entwickelt, verbirgt, dass diese in Diskurse hinein ragen, die zu den großen Themen der sechziger und frühen siebziger Jahre innerhalb und außerhalb der Planungswissenschaft geführt wurden. Dazu gehören - um grob zu etikettieren - die,Technokratiediskussion', der sogenannte ,Deutsche Positivismusstreit' und die Profilierung der Planungstheorie im Übergang von szientifischer zu kommunikativer Rationalität und damit das von Planung als politischem Handeln. Ich wurde eingeladen, diesen Kommentar zu schreiben und werde im Folgenden über einige der damaligen Beiträge berichten, die das Feld beschreiben, auf dem sich Burckhardt bewegt. Als Zeitzeuge kann ich berichten, dass Burckhardt durch seine Vernetzung und Präsenz in zahllosen Vorträgen und Veröffentlichungen die planungstheoretische Debatte jener Zeit entscheidend mitgeprägt hat. Sein Aufsatz „Wer plant die Planung?“ von 1974 formuliert besonders prägnant die Desillusionierung der Wissenschaftlichkeit von Planung und platziert sie im politischen Raum (Burckhardt 2017 [1974]: 114). Die Wende hin zur einer Perspektive auf das Netz der Akteur_innen und ihre Interessenlagen gehört zu den damals zukunftsweisenden Leistungen.

1974 hatte die Planungstheorie, das engere Gebiet also, in das sich Burckhardts „Wer plant die Planung?“ einbettet, bereits eine Strecke mit besonders dramatischen Wendepunkten hinter sich. Das ist nicht verwunderlich. Zum einen steckte das, was sich unter Planungs-/Entwurfs-/Designtheorie versammelte, noch in den Geburtswehen. Sie hatte keine so lange Geschichte hinter sich wie andere Disziplinen, etwa die Soziologie oder die Ökonomie, die - merkbar - Burckhardts Herkunftsdisziplinen sind. Zum anderen wirkten die Impulse, die sich um 1968 entwickelten, jener symbolisch aufgeladenen Jahreszahl von axialem Rang für die Diskurse davor und danach, auch auf die Planungswissenschaften.

Schon mit der Formulierung des Titels stößt Burckhardt listig das Fenster auf für eine Perspektive auf die Abhängigkeit der Planung von ihren Akteur_innen und denen, die hinter den Planer_innen als weitere Akteur_innen stehen, und auf den Kontext von Rollenbeschränkung 
und politischem Einfluss. Mit seinem ersten erläuternden Satz positioniert er sich im Lager derer, die Planung nicht als fachlich isolierbare Disziplin, sondern als Tätigkeit in gesellschaftlichem Kontext sehen, ,... daß sie bedingt ist durch die Politik, daß sie aufgehängt ist in einem sozialen System." (Burckhardt 2017 [1974]: 105) Und nach zwei Seiten Lektüre hat er das kategoriale Feld abgesteckt, in dem das Planen zu verankern sei. Kernpunkte sind die Problemformulierung als bereits für die Lösungsstrategie wirksame Entscheidung, die Nicht-Objektivität der Entscheidung, die Bindung an (Wahl-)Erfolge der entscheidenden Akteur_innen, die Kritik am (Demokratie-)Modell in Bezug auf seine Griffigkeit für Planungsentscheidungen, die Infragestellung der Unabhängigkeit der Fachleute und deren Verwebung in Macht und Politik, die Fragwürdigkeit eines ,formalistischen Demokratiebildes'. Konkreter fällt der Begriff,Lobby', der die Stadtplanung unheilige Allianzen mit Investor_innen und Baugewerbe eingehen lässt.

Man kann den Blick auf die Mitstreiter_innen in diesem Diskurs mit Horst Rittel beginnen, mit dem Burckhardt seit 1959, wo sie sich an der Hochschule für Gestaltung (HfG) Ulm begegneten, eine lebenslange Freundschaft verband, und dem er bescheinigte, eine ganze Generation von Planer_innen geprägt zu haben. Dessen Befunde von 1969/1973, dass alles Planen politisch sei (mündlich in Vorlesungen, vgl. Rittel 1992: 35) oder, im Rahmen seiner berühmten ,wicked problems', dass es keine definitive Formulierung eines Planungsproblems gäbe, und dass die gewählte Erklärung des Problems die Lösung bestimme, zeugen von der gleichen Position. Aber es ist nicht nur dieser ebenfalls denkmächtige Planungstheoretiker, es sind auch Autor_ innen aus der Soziologie, Sozialphilosophie und Politologie, deren Beiträge den Hintergrund für Burckhardts Einlassungen mit geformt haben.

\section{Planung ist politisches Handeln}

Wenn Burckhardt (S. 109) den Widerspruch zwischen dem formalen und dem effektiven Aufbau der planenden Organisation beschreibt, so sieht er die Entscheidungsmacht aufgrund der besseren Informationslage von Verwaltung zu Regierung zu Parlament abnehmen statt, wie im formalen Modell, umgekehrt. Die Entscheidung im Parlament ist dann, zeremonialisiert'. Was Edelman (1964) in den USA noch formal-kritisch als ,symbolischen Gebrauch von Politik' charakterisierte, wurde in Deutschland Gegenstand einer viel grundsätzlicheren kritischen Theorie staatlicher Planung, welche die Unzulänglichkeiten des Planungsapparates zu erklären versucht. Dabei gab es, von Frieder Naschold (1972) grob eingeteilt, zwei Lager: ein polit-ökonomisches und ein handlungs-/entscheidungstheoretisches. Das politökonomische sah die Erklärung von Dysfunktionalitäten in einer grundsätzlichen Abhängigkeit der Politik von der Ökonomie, von ihren mächtigen Akteur_innen, und grundsätzlicher noch in dem ungelösten Widerspruch zwischen Kapital und Lohnarbeit. Der Staat übernimmt die Aufgabe Konflikte auszuregeln, sichert im Prinzip aber die Funktionsbedingungen einer kapitalistischen Wirtschaft, so Autoren wie Altvater (1972) und von Flatow/Huisken (1973). Die darin erscheinenden zentralstaatlichen Machtverhältnisse schlagen, wie Grauhan (1969) bemerkt, dann auch im Handeln der planenden Verwaltung auf kommunaler Ebene durch. Für einen Teil dieser Theoretiker war die 
Strategie der Änderung des Missstandes die Vorbereitung der Revolution, nach der dann radikaldemokratische Modelle bessere Pläne hervorbringen würden. Diese Position wurde auch geteilt von der Berliner Fraktion der von Burckhard erwähnten (S. 107) Architekturstudent_innen und kam in der damals berühmten „Planer-Flugschrift“ von 1968 zum Ausdruck (Autorenkollektiv der Fachschaft Architektur, TU Berlin, 1968). Die Verwissenschaftlichung der Planung solle nicht der Aufrechterhaltung der derzeitigen Herrschaftsstrukturen dienen, sondern ihrer Änderung beziehungsweise nach ihrer Änderung den dann Herrschenden.

Parallel gab es das handlungs- und entscheidungstheoretische Lager, das einem pluralistischen Systemkonzept folgte, in dem der Mechanismus miteinander konkurrierender Gruppen in Aushandlungsprozessen ein „Optimum kollektiver Rationalität" erzeugen kann, wie es Naschold (1973) in „Gesellschaftsreform und politische Planung“ beschreibt. Selbst wenn ein Ungleichgewicht der Interessendurchsetzung zu konstatieren ist, so können Fehler im irrationalen Verhalten von Gruppen oder der Verwaltung lokalisiert und korrigiert werden; es gebe ausreichend Möglichkeiten der Reform der Missstände, so Sontheimer (1971) in seinen Grundzügen des politischen Systems der Bundesrepublik Deutschland und zeitgleich Scharpf (1971) in Planung als politischer Prozess. Zu den Mitteln der Verbesserung gehören neue Planungs- und Informationstechniken und auch neue Formen der Beteiligung.

Eine besondere Position nahm Offe (1972) in Strukturprobleme des kapitalistischen Staates und (1969) in „Politische Herrschaft und Klassenstrukturen“ ein. Ausgehend von der These, dass am Output des Entscheidungssystems ablesbar sei, wie (un-)demokratisch der Staat ist, stellt er fest, dass wichtige Fragen gar nicht auf die Agenda kommen (siehe Burckhardt 2017 [1974]: 105) und dass einseitige Interessen, die mit dem Profit aus Boden und Kapital zu tun haben, sich durchsetzen. Generell, so seine ,Disparitätentheorie', stellt er fest, dass Politikbereiche wie Wirtschaft, Finanzen und Militär höheres Gewicht haben als solche, die sich mit Wohnen, Gesundheit oder Bildung befassen.

Burckhardt steht als Planungstheoretiker auf der Seite dieser Gruppe der kritischen Theorie. Wenn er in einem eigenen Abschnitt zu ,kollektiven Entscheidungen' die Funktionsweise des Systems kritisiert, nimmt er zudem innerorganisatorische Verhaltensgesetze ins Visier, wie sie auch in den USA von March/Simon (1958) in Organizations, von Simon (1947) in Adminstrative Behaviour und von Olson (1965) in The Logic of Collective Action untersucht wurden.

Zum anderen richtet Burckhardt seine Kritik auf die Funktionsweise des Demokratiemodells, das in eine Umkehrung des intendierten Entscheidungsmechanismus ausartet (s. o.). Wenn er auf den ,großen Teil der modernen Entscheidungsforschung" (S. 106) hinweist, ordnet er sich Gesinnungsgenossen aus der Riege der kritischen Politologen der frühen 7oer zu. Zu nennen sind Naschold, Väth, Scharpf, Hirsch, Horn, Massing oder Grauhan, die meisten versammelt in dem Band Politikwissenschaft (Kress/Senghaas 1971). Sie trugen die Defizitanalyse des Modells demokratischer Entscheidungsfindung in seiner praktischen Ausformung vor. Wahlen werden nach personalisierten Profilkämpfen zu Entscheidungen über Führungsfiguren, weniger zu Entscheidungen über konkurrierende Programme. Propaganda und Marketing bis hin zur Manipulation überwiegen die Argumentation. Ohnehin kann 
sich der Wahlakt nicht auf die Einzelprobleme beziehen, die im Laufe einer Wahlperiode auftauchen. Besonders kritisiert wird auch die von Burckhardt benannte widersinnige Umkehrung des Machtgefälles von Parlament über Regierung zu Verwaltung. Parlamentarier seien oft überfordert von der Komplexität der Materie; die Zielkonflikte, die erst bei Ausarbeitung und Vollzug der Vorgaben von oben auf der Verwaltungsebene auftauchen, werden dort entschieden, so Grauhan (1969) in „Zur Struktur der planenden Verwaltung“. Die Verwaltung macht Vorlagen für das Parlament. Referent_innen arbeiten die Gesetzentwürfe aus. Da werden Zielalternativen im vorparlamentarischen Raum - oft im Austausch mit Lobbyist_innen - ausgeschieden, sodass nur eine akzeptable Lösung verbleibt. Die Verwaltung gewinnt aus den Vollzugsdaten relevantes Wissen. Das Parlament wird abhängig, die Verwaltung übernimmt indirekt Regierungsarbeit und nimmt dadurch an der politischen Führung teil, so Hirsch (1972) in seinem Aufsatz „Ansätze einer Regierungslehre“. Kritik, zum Beispiel von Olson (1965) und von Offe (1972), richtet sich überdies auf die feststellbare Asymmetrie der Einflusschancen zwischen bevorteilten (organisierten) Interessenverbänden und benachteiligten (unorganisierten) Betroffenengruppen, welche den größeren Anteil in der Gesellschaft ausmachen. Burckhardt nennt sie „die Stimme der Verplanten“ (S. 111).

\section{Gesamtplan vs. Durchwurschteln}

Es gibt noch eine andere Dimension des planerischen Handelns, die in Burckhardts Text auftaucht. Er spricht vom „Stolpern von Maßnahme zu Maßnahme“ einerseits und „strategischem Vorgehen“ andererseits, „wie es dem Systemcharakter der Stadt angemessen wäre" (S. 113). Hier mischen sich zwei Debatten. Die eine bezieht sich auf das Konzept der Gesamtplanung, in den USA als ,comprehensive“ bezeichnet, gegenüber dem des ,Durchwurschtelns', was als ,muddling through ' durch den Aufsatz von Lindblom (1959) theoretische Gestalt verliehen bekam. Es sei realistischer als ohnehin nicht durchsetzbare Gesamtpläne und entwickle in der Addition der Schritte seine eigene Rationalität. Wesentliche Referenz in dieser Debatte ist Popper (1965), der Vater des ,Inkrementalismus', der im ,Elend des Historizismus der ,Politik der kleinen Schritte' den Vorzug vor Gesamtlösungen gibt, die Gefahr laufen, totalitären Charakter zu entwickeln. Festgehalten werden kann aber auch eine Erkenntnis von Braybrooke/Lindblom (1963) als Vertretern des Inkrementalismus, dass sich Ziele und Mittel wechselseitig bedingen.

\section{Systemtheorie}

Der Aspekt jedoch, den Burckhardt anspricht, ist ein anderer. Er setzt das Stolpern von Maßnahme zu Maßnahme in Bezug zu der Unfähigkeit, der Komplexität des Systems Stadt und der Komplexität seiner Probleme (mit adäquaten Lösungen) gerecht zu werden. Er favorisiert hier das Konzept der Systemtheorie, obgleich es in den damaligen Debatten als Versuch unangemessener Verwissenschaftlichung des Planergeschäftes kritisiert wurde. (Siehe die Kritik am Einsatz kybernetischer Modelle zur ,Selbstregelung“ von Systemen als Objekte der Planung durch Kade/Hujer 1972). Er sieht aber das Potential der systemischen Herangehensweise. Unter anderem 
auch von Rittel transportiert, der mit Churchman (1968: The Systems Approach) in Berkeley kooperierte, versprach der Ansatz, die Komplexität eines Planungsproblems, beziehungsweise der Materie, auf die sich Planung bezieht, (eine Stadt z. B.), gerade nicht unzulässig zu vereinfachen. Er bot eine Möglichkeit, sich der relevanten Elemente, ihrer Wechselbeziehungen und der möglichen Folgen von erwogenen Maßnahmen im Wirkungsgefüge zu vergewissern, bevor gehandelt wird - eine der fundamentalen Kompetenzen von Planer_innen.

Nebenbei setzt Burckhardt noch einen Seitenhieb auf die Ausbildung der Architekt_innen, die in Deutschland Stadtplanung betreiben; sie seien wegen ihres Trainings in Komplexitätsreduktion und intuitiver Lösungstaktik für eine solche Aufgabe gar nicht geeignet. Die von Burckhardt gelobten Initiativen der Architekturstudent_innen vor und nach den 68er Jahren griffen tatsächlich diese Selbstbegrenzung des Fachs auf und erreichten, besonders erfolgreich in der Stuttgarter Fraktion (der ich damals angehörte), die Einführung von Fächern wie Soziologie und Bauökonomie, ja der Planungstheorie selbst, in denen ein kritischer Diskurs über die eigene Tätigkeit überhaupt erst stattfinden konnte. Diese Initiative ebnete auch Horst Rittel 1972 den Weg von Berkeley nach Stuttgart. Die ihm als Mathematiker und theoretischem Physiker zugeschriebene Position, er wolle Planung zur Wissenschaft machen, verkennt seinen wesentlichen Beitrag. Selbst wenn er in Kenntnis der Techniken des Operation Research und des Systemansatzes, wie ihn Churchman in Berkeley vertrat, eine breite Palette von Methoden anbot, um, wo immer es sinnvoll war, den Planungsprozess rational zu stützen, ist seine Hauptbotschaft in der Charakterisierung von Planungsproblemen als ,wicked problems ' formuliert. In seinem zusammen mit Webber nach einem 1969 in Boston gehaltenen Vortrag verfassten Aufsatz (Rittel/Webber 1973) über die „Dilemmas in a General Theory of Planning“ zählt er Merkmale von Planungsproblemen auf, die sie dramatisch und grundsätzlich von solchen der Ingenieure und Wissenschaftler unterscheiden. Um die Differenz zu betonen, hat Rittel diesen Ansatz auch ,System Theory of the Second Generation' genannt.

Einsichten, wie sie in den Eigenschaften der ,Bösartigkeit‘ (wickedness) ausformuliert werden, sind Burckhardt nicht fremd. Es ist schwer zu sagen, welcher Art die wechselseitige Befruchtung von Burckhardt und Rittel war. Sie haben sich gekannt, besucht und ausgetauscht. Immerhin ist die Ähnlichkeit ihrer Erkenntnisse frappierend. Wenn Burckhardt etwa die „determinierende Kraft [...] in der Benennung des Übelstandes“ (Burckhardt 2017 [1974]: 109) sieht, wodurch die „Art seiner Bekämpfung“ (ebd.) festgelegt wird, so entspricht es den Eigenschaften bösartiger Probleme, dass die Formulierung des Problems beziehungsweise seine Erklärung die Lösung festlegt. Oder wenn Burckhardt sagt, dass die Bekämpfung einer Misere (am Bahnhofsplatz, S. 109) besser woanders (am Stadtrand) erfolgen solle, wo es nämlich erzeugt wird, formuliert Rittel, dass jedes Problem als Symptom eines anderen gesehen werden kann, dessen Lösung angemessen wäre. Schreibt Burckhardt, „Verantwortung ist ein ungedeckter Scheck, da Fehlplanungen nicht rückgängig gemacht werden“ (S. 113) können, konstatiert Rittel, der Planer habe kein Recht darauf Unrecht zu haben. Es ist müßig, zu spekulieren, wer da der Vater eines Gedankens war. Sie waren unabhängige Denker, die sich trafen. Sie waren Brüder im Geiste. 


\section{Die Technokratie-Diskussion}

Die Auseinandersetzung um das Verhältnis von Wissenschaft und urteilendem ,politischem' Entscheiden berührt eines der großen Themen der sechziger Jahre. Was bei Burckhardt in dem Kontrapaar „Politik“ versus „Planung“ (S. 106) oder am Schluss seines Aufsatzes (S. 114) als „technische Planung“versus „Demokratisierung“ auftaucht, verweist auf eine Debatte, die zu der Zeit noch nicht für alle entschieden war, die aber auf vielen Ebenen seit über einem Jahrzehnt geführt wurde. Schelsky (1961) eröffnete die sogenannte ,Technokratie-Diskussion' mit der Vision zukünftiger Entscheidungsmechanismen, bei denen verfügbare Mittel sachzwangartig über den Fortschritt der Gesellschaft bestimmen. An Menschen gebundene Urteile, die Bedürfnisse und Wertorientierung ausdrücken und in demokratische Prozeduren eingebunden sind, werden dadurch überflüssig. Die Planer_in ist als Expert_in Teil und Ausführende_r eines technisch-wissenschaftlichen, in Bürokratie gegossenen Apparates ohne ethische Verantwortung. Neben anderen Opponenten wie Barth (1961) mit seiner berühmten Reaktion „Helmut Schelskys technischer Staat“ formulierte Habermas (1968) die später am meisten referenzierte Gegenposition in Technik und Wissenschaft als Ideologie. Habermas sieht den, auch durch den Zwang der Instrumente, nicht abgedeckten Entscheidungsrest einem ,dezisionistischen' Akt überlassen. Der Herrschaft der Instrumente stellt er die Vorrangigkeit der in der ,Lebenswelt` vorhandenen Bedürfnisse der Menschen gegenüber. Gegen die Webersche ,Zweckrationalität' oder instrumentelle Rationalität, in der bei (von wem?) vorgegebenen Zielen nur der Einsatz der Mittel Gegenstand der Überlegung ist, steht eine andere Rationalität, die sich gerade aufZiele, auf die handlungsleitenden Normen bezieht. Die,kommunikative Rationalität' beruht auf den Beiträgen der Beteiligten, insbesondere der handelnden Bürger_ innen, die ihre Positionen und Argumente in einen Diskurs einbringen, der idealerweise ,öffentlich, uneingeschränkt und herrschaftsfrei‘ sein solle.

Später entwickelte sich eine Diskussion darüber, ob jener von Habermas (1973) in Legitimationsprobleme im Spätkapitalismus erklärtermaßen ,antifaktisch' postulierte herrschaftsfreie Raum möglich sei. Natürlich nicht. Die Sprechsituation ist ,ideal'. Es ist ein als normativ leitend konstruiertes Setting, auch wenn - und gerade weil - die Realität mit ihrem Gebrauch ungerechtfertigter Macht widerständig ist.

\section{Von der ,Scientific Method' zum argumentativen Modell oder der Kommunikativen Rationalität}

Der Ausgang der unter Soziolog_innen, Sozialphilosoph_innen und Politolog_innen geführten ,Technokratiediskussion` bestätigt für die Disziplin der Planer_innen, dass ihr Handeln politisch ist. Was Scharpf (1971) in Planung als Politischer Prozess als Politologe formuliert und Rittel (1973) als Planungstheoretiker in „Dilemmas in a General Theory of Planning“ erschlossen hat, ändert ein Paradigma der Rationalität, das bis dahin die Planungstheorie geleitet hatte. In ihren Anfängen bezog sie sich auf den Operations Research mit seinen im Militär und später in Wirtschaftsunternehmen entwickelten Methoden. Im Sinne der Weberschen Zweckrationalität bestand ihre Leistung 
in der ,optimalen Allokation von Ressourcen' bei gegebenen Zielen. (Das einfache Unternehmensziel ist das allen ökonomischen Handelns: maximaler Output bei minimalem Input.) Sie firmierte auch als ,scientifische Rationalität‘, in Anlehnung etwa an Ackoffs (1962) Scientific Method, weil man davon überzeugt war, mit wissenschaftlichen Methoden in der Planung die gleiche Evidenz für die Richtigkeit einer Planungsmaßnahme erzeugen zu können wie die Wissenschaft in ihrem Bereich für Fakten und Erklärungen. Am Versagen dieser Versuche setzt der erwähnte Aufsatz von Rittel und Webber (1973) an. Er erklärt es mit der unterschiedlichen Art der Probleme, mit denen es Wissenschaftler und Planer im Prinzip zu tun haben. Der entscheidende Aspekt, die Urteilsabhängigkeit planerischer Probleme in einem Kontext zunehmend pluralistischer Gesellschaften, führt zur Methode des sorgfältigen Argumentierens auf dem Weg zur Entwicklung einer Lösung. Rittel hat es in anderen Aufsätzen als ,Argumentatives Modell' formuliert (vgl. Rittel 2013). Es kann als eine zeitgleich entwickelte instrumentelle Version des kommunikativen Modells Habermasscher Prägung gesehen werden. Aber erst dessen Rezeption in den USA hat zum sogenannten ,argumentativen' oder ,communicative turn' (Healey 1992) geführt. Für Burckhardt war der Gedanke, auch durch seine Freundschaft mit Rittel, 1974 längst präsent. Heute noch ist Rittels Etikett der ,wicked Problems oder ,bösartigen Probleme' unzitiertes Label für das vom wissenschaftlichen zu unterscheidende, radikal andere Profil planerischer Probleme.

\section{Der Positivismusstreit}

Es liest sich wie ein ärgerlicher Seitenhieb, wenn Burckhardt zynisch über den Beitrag der Soziolog_innen zum Aufspüren der Zustimmung der Betroffenen spricht und bei der Kritik der empirisch erlangten Ergebnisse die „Dissonanzreduktion“ (S. 111) anführt. In dem Hinweis auf die fragwürdige „Aussagekraft von Befragungen“(ebd.), oder wenn er vom „Anschein der Aufklärung“ (ebd.) spricht, dann blitzt da ein anderer großer Diskurs der Jahre vor 1974 auf, in dem Burckhardt als Soziologe zu Hause ist - die Debatte, die als ,deutscher Positivismusstreit ' in die Geschichte einging. Im gleichnamigen Sammelband von Adorno et. al (1972) greifen Adorno und Habermas als Vertreter der Frankfurter Schule die Hauptfeinde Popper und Albert als Vertreter des Positivismus an. Ihr Vertrauen auf die an den Methoden der Naturwissenschaften orientierte Empirie zur Beschaffung von Evidenz für Hypothesen stößt an Grenzen. Wenn der aufzuspürende Sachverhalt, der erfragt werden soll, nicht selbst Teil der reflektierbaren Einstellung eines zu Befragenden ist, sondern - so die Annahme der Frankfurter - zum Beispiel durch eine Bewusstseinsindustrie, durch „Methoden, mit denen man sich Zustimmung schafft" (S. 111), beeinflusst wird, dann ist eine analytische Theoriebildung zur Auffindung der Wahrheit geeigneter. Dann sollten so Habermas in der Entgegnung auf Albert (s. Adorno et al. 1972) - auf ihre Gültigkeit und nach Kriterien überprüfbare Argumente Elemente der Evidenzbeschaffung sein dürfen. In diesem Sinne spricht Burckhardt von der Art, wie der Einzelne die Wirklichkeit zu sehen vermeint, als einer Folge seiner Erziehung in der Gesellschaft, von seiner „soziale[n] Konstruktion“ (S. 112), eine Formulierung, mit der er sich im Lager der Kritischen Theorie der Frankfurter Schule positioniert. 
Wie auch immer Spätere diesen Streit für sich als entschieden angenommen haben mögen, so hat er, wie bei Burckhardt abzulesen, die Fähigkeit zur Kritik an der Empirie als Methode, die „Stimme der Verplanten“ (S. 111) zu hören, gestärkt. Wieder ist eine Bastion der Wissenschaftlichkeit im Planungsgeschäft brüchig geworden, weil sich auch hier der politische Charakter von Planung zeigt. Mit dem Gebrauch von Macht vereinnahmt der politisch-administrative Apparat nicht nur die Expert_innen, die er beauftragt und bezahlt. Aus der Erkenntnis, dass die Methoden der Zustimmungsbeschaffung wie ,Information“ und ,Beratung' missbraucht werden, zieht Burckhardt den Schluss, weitere Schritte auf dem Weg der Beteiligung zu gehen. In seinem Schlussabsatz resümiert er nochmals, dass Planung nicht autonom sei (S. 113). Er sieht das Fortschreiten des Partizipationsgedankens, dessen weitere Ausdehnung sich erst nach 1974 zeigte. Wenn er die Rolle der Planungstheorie als Lieferantin von Objektivität angreift, verfolgt er ein Bild, das sich noch lange halten würde, aber zu dessen Dekonstruktion er selbst beiträgt. Die Politisierung, die kommunikative Einbindung der Beteiligten mit dem Zweck Machtgefälle abzubauen, die Entlarvung des Gebrauchs von Macht mit ihren Techniken selbst, der Aufbau von Gegenmacht durch Partizipation, hatte 1974 bereits begonnen und Burckhardt trug dazu bei, ihr in Zukunft mehr Geltung zu verschaffen.

\section{Autor_innen}

Wolf Reuter ist Design- und Planungstheoretiker, sowie Romanautor. Er forscht zu Planungsmethoden, Argumentativer Planung und zur Macht der Planer_innen und Architekt_innen. iwereute@iwe.uni-stuttgart.de

\section{Literatur}

Ackoff, Russel L. (1962): Scientific Method: Optimizing Applied Research Decisions. New York/London: John Wiley \& Sons.

Adorno, Theodor W. / Dahrendorf, Ralf / Pilot, Harald / Albert, Hans / Habermas, Jürgen / Popper, Karl R. (1972): Der Positivismusstreit in der deutschen Soziologie. Darmstadt/ Neuwied: Luchterhand.

Altvater, Elmar (1972): Zu einigen Problemen des Staatsinterventionismus. In: Probleme des Klassenkampfes 3, 1-53.

Autorenkollektiv der Fachschaft Architektur, TU Berlin (1968): Planer-Flugschrift: Ein Beitrag zur Planerausbildung. In: Stadtbauwelt 20, 1499-1504.

Barth, Hans Paul (1961): Helmut Schelskys technischer Staat. In: Atomzeitalter 4: 195-201.

Braybrooke, David / Lindblom, Charles E. (1963): A Strategy of Decision. New York: The Free Press.

Burckhardt, Lucius (2017 [1974]): Wer plant die Planung?. In: s u b $\backslash$ u r b a n . zeitschrift für kritische stadtforschung, 5, 1-2, 105-114.

Churchman, C. West (1968): The Systems Approach. New York: Delacorte Press.

Edelman, Murray Jacob (1964): The Symbolic Uses of Politics. Illinois: University of Illinois Press.

Grauhan, Rolf-Richard (1969): Zur Struktur der planenden Verwaltung. In: Stadtbauwelt 22, $132-137$.

Habermas, Jürgen (1968): Technik und Wissenschaft als „Ideologie“. Frankfurt am Main: Suhrkamp. 
Habermas, Jürgen (1973): Legitimationsprobleme im Spätkapitalismus. Frankfurt am Main: Suhrkamp.

Healey, Patsy (1992): Planning through debate: The communicative turn in planning theory. In: The Town Planning Review 63/2, 143-162.

Hirsch, Joachim (1972): Ansätze einer Regierungslehre. In: Gisela Kress / Dieter Senghaas (Hg.), Politikwissenschaft. Eine Einführung in ihre Probleme. Frankfurt am Main: Fischer Taschenbuch Verlag, 269-285.

Kade, Gerhard / Hujer, Reinhard (1972): Planung der kleinen Schritte und Politik des „Status quo“. Zur systemerhaltenden Funktion von Planungskonzepten. In: Gerhard Fehl / Mark Fester / Nikolaus Kuhnert (Hg.), Planung und Information. Materialien zur Planungsforschung. Gütersloh: Bertelsmann Fachverlag, 167-179.

Kress, Gisela / Dieter Senghaas (Hg.) (1971): Politikwissenschaft. Eine Einführung in ihre Probleme. Frankfurt am Main: Fischer Taschenbuch Verlag.

Lindblom, Charles E. (1959): The science of „muddling through“. In: Public Administration Review 19/2, 79-88.

March, James G. / Simon, Herbert A. (1958): Organizations. New York: Wiley.

Naschold, Frieder (1972): Systemsteuerung. Einführung in die moderne politische Theorie. Teil II. Stuttgart: Kohlhammer.

Naschold, Frieder (1973): Gesellschaftsreform und politische Planung. In: Frieder Naschold / Werner Väth (Hg.): Politische Planungssysteme. Opladen: Westdeutscher Verlag, 59-98.

Offe, Claus (1972): Strukturprobleme des kapitalistischen Staates. Frankfurt am Main: Suhrkamp.

Olson, Mancur (1965): The Logic of Collective Action: Public Goods and the Theory of Groups. Cambridge (Massachusetts): Harvard University Press.

Popper, Karl (1965): Das Elend des Historizismus. Tübingen: Mohr.

Rittel, Horst / Webber, Melvin (1973): Dilemmas in a general theory of planning. In: Policy Sciences 4: 155-169.

Rittel, Horst (1992): Planen Entwerfen Design. Ausgewählte Schriften zu Theorie und Methodik. Stuttgart: Kohlhammer.

Scharpf, Fritz (1971): Planung als politischer Prozess. In: Die Verwaltung 1, 1-30.

Schelsky, Helmut (1961): Der Mensch in der wissenschaftlichen Zivilisation. Köln: Westdeutscher Verlag.

Simon, Herbert A. (1947): Administrative Behaviour: A Study of Decision-making Processes in Administrative Organizations. New York: Macmillan.

Sontheimer, Kurt (1971): Grundzüge des politischen Systems der Bundesrepublik Deutschland. München: Piper.

Von Flatow, Sybille / Huisken, Freerk (1973): Zum Problem der Ableitung des bürgerlichen Staates. In: Probleme des Klassenkampfes 7, 83-153. 
